\title{
Hormetic Effects of Thiophanate-Methyl in Multiple Isolates of Sclerotinia homoeocarpa
}

Sumit Pradhan, Department of Entomology and Plant Pathology, Oklahoma State University, Stillwater; Lee Miller, University of Missouri, Division of Plant Sciences, Columbia; Vanessa Marcillo and Alma R. Koch, GIMA Research Group, Department of Live Sciences and Agriculture, Universidad de las Fuerzas Armadas-ESPE, Sangolquí, Ecuador; Nathalia Graf Grachet, Julio E. Molineros, Nathan R. Walker, and Hassan Melouk, Department of Entomology and Plant Pathology, Oklahoma State University, Stillwater; and Carla D. Garzon, ${ }^{\dagger}$ Department of Entomology and Plant Pathology, Oklahoma State University, Stillwater, and GIMA Research Group, Department of Live Sciences and Agriculture, Universidad de las Fuerzas Armadas-ESPE, Sangolquí, Ecuador

\begin{abstract}
Twenty-eight isolates of Sclerotinia homoeocarpa, causal agent of dollar spot disease in turf, were assessed for fungicide hormesis at sublethal concentrations of thiophanate-methyl (T-methyl). Each isolate was grown in corn meal agar amended with 11 concentrations of T-methyl $(30,500$ to $0.047 \mu \mathrm{g} / \mathrm{liter})$, and the area of mycelial growth was determined relative to the control. Three replicates were used per concentration, and the experiment was repeated three to five times for each isolate. Reference isolates $\left(\mathrm{EC}_{50}>20 \mu \mathrm{g} / \mathrm{liter}\right.$ ), with no prior history of T-methyl exposure, were highly sensitive and not stimulated by low

doses. Likewise, no stimulation was observed in two highly sensitive isolates $\left(\mathrm{EC}_{50}>30 \mu \mathrm{g} / \mathrm{liter}\right)$ that had been preconditioned by exposure to T-methyl, or in four T-methyl-tolerant isolates. Seventeen (81\%) preconditioned T-methyl-tolerant isolates $\left(\mathrm{EC}_{50}=294\right.$ to $\left.1,550 \mu \mathrm{g} / \mathrm{liter}\right)$ had statistically significant growth stimulation, in the range of 2.8 to $19.7 \%$ relative to the control. These results support that hormesis (low-dose stimulation, high-dose inhibition) is a common dose response in preconditioned $S$. homoeocarpa, particularly in response to subtoxic doses of T-methyl.
\end{abstract}

Sclerotinia homoeocarpa F.T. Bennett is a plant pathogenic ascomycete that causes disease on more than 40 plant hosts, the majority of which belong to the grass family Poaceae (Bennett 1937; Monteith and Dahl 1932). The disease, known as dollar spot, is most economically important on creeping bentgrass (Agrostis stolonifera L.), which is extensively used on golf course fairways and putting greens (Smiley et al. 2005). Management with fungicides is the principal method used to control dollar spot (Walsh et al. 1999). Although many fungicides with different modes of action are commercially available, the frequent and repeated use of fungicides throughout the growing season has resulted in the development of fungal isolates that are resistant to almost all fungicide groups (Cole et al. 1968; Detweiler et al. 1983; Golembiewski et al. 1995; Hsiang et al. 1997; Miller et al. 2002; Nicholson et al. 1971; Warren et al. 1974).

Because fungicides are frequently used every year to control dollar spot in golf courses, it is often one of the most expensive diseases of turfgrass to control (Vargas 2005). Although repeated applications of fungicides are conducted, environmental factors such as sunlight, rainfall, and microbial decomposition of active ingredients result in fungi coming in contact with subtoxic doses of fungicides. In vitro studies revealed that exposure to subtoxic doses of fungicides in fungal and oomycete pathogens triggered increased growth, longevity, mycotoxin production, and tolerance to other fungicides (Audenaert et al. 2010; Flores and Garzon 2013; Garzon et al. 2011; Zhou et al. 2014). Such induced responses are often associated with hormesis. Hormesis is a toxicological concept characterized by stimulatory effects on the metabolism of an organism resulting from exposure to subinhibitory doses of a stressor (chemical or radiation) that is toxic

${ }^{\dagger}$ Corresponding author: Carla D. Garzon; E-mail: carla.garzon@okstate.edu

Funding: This research was funded by the Oklahoma Agricultural Experiment Station (grant no. OKL 02859).

*The $\boldsymbol{e}$-Xtra logo stands for "electronic extra" and indicates that two supplementary figures are published online.

Accepted for publication 4 July 2018.

@ 2019 The American Phytopathological Society at higher doses (Calabrese and Baldwin 2002). Stimulatory effects in plant pathogens resulting from exposure to subinhibitory doses of fungicides have been described as "fungicide hormesis" (Garzon et al. 2011). Initial overcorrection to a disruption in homeostasis (Stebbing 1982) and direct stimulation (Calabrese 2013; Szabadi 1977) are often considered the two possible mechanisms underlying hormesis.

There are few cases of fungicide hormesis reported for economically important fungal plant pathogens in recent phytopathological literature. Improved germination for some thiabendazole-resistant isolates of Penicillium expansum was reported when exposed to a subinhibitory dose of thiabendazole (Baraldi et al. 2003). Audenaert et al. (2010) showed that exposure to low doses of prothioconazole led to a higher production of deoxynivalenol in vitro and in planta by Fusarium graminearum. Significant radial growth stimulation was observed in Lyophyllum palustre and Sclerotinia sclerotiorum when exposed to sublethal doses of propamocarb and dimethachlon, respectively (Landry et al. 2011; Zhou et al. 2014). Zhou et al. (2014) also found increased virulence when fungi were evaluated on detached oilseed rape plants after treatment of dimethachlon at a concentration of $2 \mu \mathrm{g} / \mathrm{ml}$. Growth stimulation after exposure to low doses of fungicides was reported for several oomycete pathogens, including Phytophthora infestans (Zhang et al. 1997), Phytophthora undulata (Kato et al. 1990), and Pythium aphanidermatum (Flores and Garzon 2013; Garzón et al. 2011). These findings highlight the importance of understanding the nature of fungicide hormesis and its potential consequences on plant health.

The objective of this study was to investigate the potential for hormesis in $S$. homoeocarpa collected from various sites in the United States with varying levels of fungicide exposure and sensitivity.

\section{Materials and Methods}

Isolates of $S$. homoeocarpa. Twenty-eight $S$. homoeocarpa isolates (Table 1) obtained from creeping bentgrass (A. stolonifera L.) putting greens or fairways were obtained for evaluation (Ma and Tredway 2013; Putman et al. 2011). Isolates were stored on colonized filter paper dried and stored at $-80^{\circ} \mathrm{C}$. Five isolates (LWC27, LWC5, LWC10, PST4, and VGC5) were collected from sites with little to no previous history of fungicide use and were chosen as reference isolates for comparison with other isolates (Ma and Tredway 2013; Putman et al. 2011). The remaining 23 isolates were collected 
from golf courses with routine fungicide use in the northeast and southeastern United States. Although these isolates have known exposure history to fungicides, and their sensitivity levels to demethylation inhibitor fungicides has been previously characterized (Ma and Tredway 2013), their specific history of benzimidazole exposure was undetermined. Preliminary tests indicated that all of the isolates included in this study were sensitive to T-methyl $\left(\mathrm{EC}_{50}<1,000 \mu \mathrm{g}\right.$ of active ingredient [a.i.] per milliliter) (Jo et al. 2008). This represented a challenge, because even though our previous research demonstrated that hormesis is also present in fungicide-sensitive isolates, stimulatory doses in those isolates tend to be at extremely low concentrations, and preparing fungicide solutions at those concentrations can be difficult to achieve with precision.

T-methyl preconditioning. To obtain isolates with some level of T-methyl tolerance, the 23 isolates with previous fungicide history were preconditioned by exposure to a low dose of T-methyl. Preconditioning consists in exposing the specimen under study to low doses of a stressor, after which the organism develops tolerance to higher doses (Calabrese et al. 2007; Lu et al. 2018). In this study, a plug of mycelium of each isolate with a previous history of fungicide exposure was transferred to corn meal agar (CMA) amended with 305 parts per billion ( $\mu \mathrm{g} / \mathrm{liter})$ technical-grade T-methyl (41.25\% a.i., Cleary Chemicals, Dayton, NJ) and incubated in the dark for $72 \mathrm{~h}$ at $25^{\circ} \mathrm{C}$. These isolates were transferred 10 times to fresh T-methyl-amended growing medium (10 vegetative generations) to obtain T-methyl "preconditioned" isolates under experimental conditions. Finally, a 5-mm plug of mycelium of each preconditioned isolate was transferred to CMA to produce inoculum for each trial.
T-methyl treatments. The sensitivity of each isolate to T-methyl was determined by measuring the mycelial surface area growth on CMA amended with six concentrations of T-methyl $(0.305,3.05$, $30.5,305,3,050$, and 30,500 $\mu \mathrm{g} /$ liter), based on the minimum application rate $(30,500 \mu \mathrm{g} /$ liter $)$ recommended on the label $(73.23 \mathrm{~kg}$ of a.i./ha). The minimum application rate value was transformed to $\mu \mathrm{g} /$ liter assuming soil bulk density of $1.2 \mathrm{~g} / \mathrm{cm}^{3}$ and an effective soil depth of $2.0 \mathrm{~cm}$ (Chen et al. 2001). All the isolates grew on CMA medium amended with fungicide at a concentration of $3.05 \mu \mathrm{g} / \mathrm{liter}$ without any significant inhibition. Therefore, the common benchmark dose (BMD: a concentration where there is no significant inhibition) for all isolates was roughly estimated to be $3.05 \mu \mathrm{g} / \mathrm{liter}$ and was used as a reference dose to assess fungicide hormesis. To identify a concentration at which growth stimulation was observed, an assay including 11 different dilutions of T-methyl (30,500, 3,050, 305, $30.5,11.94,4.75,1.89,0.75,0.3,0.119$, and $0.047 \mu \mathrm{g} /$ liter) and a fungicide-free control was conducted. Five doses were higher than BMD, and six doses were lower than BMD.

Effect of subinhibitory doses of T-methyl on fungal mycelial growth. The experimental design for detecting fungicide hormesis in this study was a modification of that used by Flores and Garzon (2013). Twenty milliliters of CMA (HiMedia Laboratories, Mumbai, India) mixed with different concentrations of T-methyl was dispensed into 9-cm-diameter Petri dishes using an accu-jet pro Pipette Controller (BrandTech Scientific, Essex, CT). A 5-mmdiameter mycelial plug from the hyphal tip region of a preconditioned isolate growing on CMA was added to each fungicideamended CMA plate. Each mycelial plug was collected from equidistant areas of the mycelium of each isolate to ensure that all the inocula used were at the same developmental stage. Each isolate was assayed

Table 1. Summary of information about Sclerotinia homoeocarpa isolates studied and results ${ }^{\mathrm{a}}$

\begin{tabular}{|c|c|c|c|c|c|c|c|c|c|}
\hline Isolate & Origin & Year & T-methyl exposure ${ }^{b}$ & $\begin{array}{c}\boldsymbol{\beta} \\
(\boldsymbol{\mu g} / \text { liter })\end{array}$ & $\begin{array}{c}\gamma \\
\text { ( } \mu \mathrm{g} / \mathrm{liter})\end{array}$ & $\begin{array}{c}\text { EC }_{50} \\
\text { ( } \mu \text { g/liter) }\end{array}$ & $\begin{array}{c}\text { TF } \\
(\mu \mathrm{g} / \text { liter })\end{array}$ & $\begin{array}{l}\text { NOAEL } \\
\text { ( } \mu \text { g/liter) }\end{array}$ & $\begin{array}{c}\text { MSD } \\
(\mu \mathrm{g} / \mathrm{liter})\end{array}$ \\
\hline LWC27 & Raleigh, NC & 2003 & Reference & 0.6729 & -0.0186 & 2.6 & $\ldots$ & $\ldots$ & $\ldots$ \\
\hline LWC5 & Raleigh, NC & 2003 & Reference & 0.7852 & -0.0502 & 4.9 & $\ldots$ & $\ldots$ & $\ldots$ \\
\hline LWC10 & Raleigh, NC & 2003 & Reference & 1.173 & -0.4481 & 7.8 & $\ldots$ & $\ldots$ & $\ldots$ \\
\hline PST4 & Rolesville, NC & 2003 & Reference & 1.2647 & -0.0281 & 11.6 & $\ldots$ & $\ldots$ & $\ldots$ \\
\hline VGC5 & Edgartown, MA & 2007 & Reference & 1.4961 & -0.5 & 11.7 & $\ldots$ & $\ldots$ & $\ldots$ \\
\hline G5 & Canal Winchester, $\mathrm{OH}$ & 2003 & Preconditioned & 4.5591 & -2.04 & 3.1 & 0.41 & $\ldots$ & $\ldots$ \\
\hline 451ShCT76 & Madison, CT & 2007 & Preconditioned & 21.987 & -0.6956 & 27.3 & 3.53 & $\ldots$ & $\ldots$ \\
\hline $\mathrm{CHCC} 10$ & North Attleboro, MA & 2007 & Preconditioned & 1.3025 & 4.7658 & 293.9 & 38.07 & 26.47 & 8.27 \\
\hline 363ShCT18 & East Falmouth, CT & 2006 & Preconditioned & 1.1967 & 46.0157 & 365.8 & 47.38 & 10.62 & 2.05 \\
\hline PhPG9 & Pinehurst, NC & 2003 & Preconditioned & 1.6017 & -0.0323 & 431.1 & 55.84 & $\ldots$ & $\ldots$ \\
\hline 557ShCT173 & Woodbridge, CT & 2007 & Preconditioned & 1.4798 & -0.0304 & 482.3 & 62.48 & $\ldots$ & $\ldots$ \\
\hline PhPG4 & Pinehurst, NC & 2003 & Preconditioned & 1.4829 & 0.4113 & 606.6 & 78.58 & 98.93 & 39.34 \\
\hline RE18G38 & Shallotte, NC & 2003 & Preconditioned & 1.715 & 0.3958 & 641.0 & 83.03 & 189.10 & 75.46 \\
\hline PhPG22 & Pinehurst, NC & 2003 & Preconditioned & 1.2318 & 10.7806 & 701.1 & 90.82 & 34.25 & 7.54 \\
\hline SO88 & Chicago, IL & 1993 & Preconditioned & 1.1483 & 97.2556 & 747.2 & 96.79 & 6.94 & 1.12 \\
\hline 725ShME & South Portland, ME & 2007 & Preconditioned & 1.3532 & 0.7022 & 796.5 & 103.18 & 87.83 & 31.78 \\
\hline RE18G8 & Shallotte, NC & 2003 & Preconditioned & 1.6549 & 0.9067 & 796.8 & 103.21 & 249.60 & 79.02 \\
\hline RE18G16 & Shallotte, NC & 2003 & Preconditioned & 1.4558 & 0.2141 & 798.3 & 103.41 & 99.81 & 41.45 \\
\hline 500ShCT123 & Coventry, CT & 2007 & Preconditioned & 1.637 & 0.3668 & 815.1 & 105.58 & 180.00 & 71.31 \\
\hline RE18G45 & Shallotte, NC & 2003 & Preconditioned & 0.7881 & -0.00367 & 822.6 & 106.56 & $\ldots$ & $\ldots$ \\
\hline RE18G35 & Shallotte, NC & 2003 & Preconditioned & 1.5843 & 0.2602 & 824.0 & 106.74 & 175.80 & 71.40 \\
\hline H127 & Springfield, $\mathrm{OH}$ & 2005 & Preconditioned & 0.8075 & -0.00385 & 828.8 & 107.36 & $\ldots$ & $\ldots$ \\
\hline A4 & Gainesville, VA & 2001 & Preconditioned & 1.2123 & 8.9181 & 964.9 & 124.99 & 35.88 & 8.21 \\
\hline A22 & Powell, OH & 2001 & Preconditioned & 1.4828 & 1.0423 & 1241.3 & 160.79 & 273.10 & 75.35 \\
\hline RE18G26 & Shallotte, NC & 2003 & Preconditioned & 1.3415 & 0.4652 & 1340.7 & 173.67 & 140.50 & 50.20 \\
\hline $\mathrm{RCC} 18 \mathrm{G} 15^{\mathrm{c}}$ & Raleigh, NC & 2003 & Preconditioned & 1.4284 & 1.1394 & 1442.7 & 186.88 & 266.70 & 70.23 \\
\hline RE18G4 & Shallotte, NC & 2003 & Preconditioned & 1.417 & 0.7101 & 1542.2 & 199.77 & 262.80 & 78.99 \\
\hline D3 & Grove City, OH & 2002 & Preconditioned & 1.1695 & 15.2964 & 1549.4 & 200.70 & 25.65 & 5.29 \\
\hline
\end{tabular}

a The modified Brain-Cousens model (Schabenberger et al. 1999) was used to determine dose parameters: $\beta$ (slope at the EC ${ }_{50}$ dose response curve), $\gamma$ (rate of growth increase at low concentrations), $\mathrm{EC}_{50}$ (the effective concentration at which $50 \%$ of response is inhibited), no-observed-adverse-effect level (NOAEL), and maximum stimulation dose (MSD) in $\mu \mathrm{g} /$ liter. The tolerance factor (TF) was calculated by dividing the $\mathrm{EC}_{50} \mathrm{value}$ of each isolate by the mean $\mathrm{EC}_{50} \mathrm{value}$ of the reference isolates $(7.72 \mu \mathrm{g} / \mathrm{liter})$. NOAEL and MSD are not reported for isolates that did not display hormetic responses.

${ }^{\mathrm{b}}$ Reference isolates had no previous exposure to thiophanate-methyl (T-methyl). Preconditioned isolates were grown on corn meal agar amended with 305 $\mu \mathrm{g} /$ liter of T-methyl for 10 vegetative generations.

${ }^{c}$ Demethylation inhibitor resistant (Ma and Tredway 2013). 
with all the T-methyl treatments. Culture plates were sealed with Parafilm and incubated in the dark at $25^{\circ} \mathrm{C}$. After $48 \mathrm{~h}$, digital images of the plates displaying mycelial growth were captured using a CanoScan $8400 \mathrm{~F}$ color image scanner (Canon USA, Melville, NY). KLONK Image Measurement 14.1.2 software (KLONK, Ringsted, Denmark) was used to measure the mycelium growth area (Pradhan et al. 2017). The software was calibrated based on a 1-cm-line scale and was used throughout the experiment to ensure uniformity. Each individual culture (one per plate) was considered as one experimental unit. Three replicates (experimental unit) were used for each concentration. Overall, 12 treatments (11 concentrations and a control) were used, with three replicates for each concentration, and the experiment was conducted 3 to 6 times for each isolate.

Data analysis. Statistical analyses for detection of significant stimulation and estimation of hormetic parameters were conducted using the modified Brain-Cousens model defined in equation 1 (Brain and Cousens 1989; Flores and Garzon 2013; Schabenberger et al. 1999). Dose parameters such as the effective concentration at which $50 \%$ of response is inhibited $\left(\mathrm{EC}_{50}\right)$, the no-observedadverse-effect level (NOAEL), and the maximum stimulation dose (MSD) were estimated using the nonlinear modeling procedure PROC-NLIN in SAS 9.2 (SAS Institute, Cary, NC).

$$
E(y / x)=\delta+(\alpha-\delta+\gamma x) /(1+\omega \exp [\beta \ln (x / \mathrm{NOAEL})])
$$

where

$$
\omega=\gamma \operatorname{NOAEL} /(\alpha-\delta)
$$

$E(y / x)$ is the expected value of the response $y$ at concentration $x ; \alpha$ is the $\%$ average mycelial growth area without concentration; $\delta$ is the $\%$ average mycelial growth area with the highest concentration; $\beta$ is the slope at the $\mathrm{EC}_{50}$ dose response curve; and $\gamma$ is the rate of growth increase at low concentrations. Dose response curves were plotted on Excel using the natural logarithms of the experimental doses (Flores and Garzon 2013). To explore the statistical relationships between $\mathrm{EC}_{50}$, TF, NOAEL, and MSD (Table 1), we estimated a linear regression between pairs, as well as Pearson's and Spearman correlation statistics in R (R Core Team 2013).

T-methyl sensitivity of preconditioned isolates. The level of sensitivity of the preconditioned isolates was scored by dividing the $\mathrm{EC}_{50}$ of each isolate by the mean of the $\mathrm{EC}_{50}$ values of all the reference isolates; these scores were referred as tolerance factor (TF) for the purpose of this study. Higher TF values represent higher levels of T-methyl tolerance. Based upon the TF value, the preconditioned isolates were further classified as sensitive $(\mathrm{TF}<10)$ and tolerant $(\mathrm{TF}$ $\geq 10$ ).

\section{Results}

Reference isolates. At a 95\% confidence interval, the estimated value of $\gamma$ for all five reference isolates (LWC27, LWC5, LWC10, PST4, and VGC5) was negative, indicating growth suppression; hence, hormetic responses were absent even at lowest T-methyl doses (Table 1; Fig. 1). Parameters $\beta$ and $\mathrm{EC}_{50}$ were determined (Table 1) by setting $\gamma=0$ and used for modeling a curve (Fig. 2). $\mathrm{EC}_{50}$ values of the five reference isolates ranged from 2.58 to $11.73 \mu \mathrm{g} /$ liter with a mean of $7.71 \mu \mathrm{g} / \mathrm{liter}$.

Preconditioned isolates. The T-methyl TF values of two preconditioned isolates, G5 and 451ShCT76, were 0.405 and 3.53, respectively, and they were classified as sensitive (Table 1). Although these isolates grew slightly more than the control in response to two fungicide treatments, neither isolate displayed significant stimulation (their estimated values for $\gamma$ were negative) at a confidence interval of $95 \%$ (Fig. 1). The model of their stimulation curve was estimated in a similar manner to that of the reference isolates, by setting $\gamma=$ 0 (Fig. 3A).

The TF value for the remaining 21 isolates was significantly higher $(\mathrm{TF}>10)$ than those of the reference and sensitive preconditioned isolates, and they were classified as T-methyl tolerant (Table 1). Parameters $\beta, \gamma$, and $\mathrm{EC}_{50}$ were calculated for each isolate. Seventeen tolerant isolates showed significant hormetic responses with positive values for $\gamma$ at 95\% confidence interval (Table 1; Fig. 1). These isolates were further analyzed to calculate the NOAEL and the MSD to estimate stimulatory responses and curve modeling (Table 1). Isolate D3 was the most T-methyl tolerant $(\mathrm{TF}=200.7)$, and $\mathrm{CHCC} 10$ was the least T-methyl tolerant $(\mathrm{TF}=38.07)$. At its $\mathrm{MSD}(5.3 \mu \mathrm{g} / \mathrm{liter})$, D3 displayed $11.7 \%$ mean stimulation with $\beta$ and $\gamma$ values of 1.17 and 15.3 , respectively. Similarly, CHCC10 showed $9.2 \%$ growth stimulation with $\beta=1.30, \gamma=4.7658$, and MSD $=8.27 \mu \mathrm{g} / \mathrm{liter}$. $\mathrm{A} 22$, with an $\mathrm{EC}_{50}$ value of $1,241.3 \mu \mathrm{g} / \mathrm{liter}(\mathrm{TF}=160.8)$, displayed the highest degree of average stimulation $(19.7 \%)$ at $\beta=1.483, \gamma=$ 1.04 , and MSD $=75.35 \mu \mathrm{g} /$ liter. A wide range of stimulation was found among isolates at different concentrations, having hormetic responses ( $\gamma=$ positive) that ranged from 2.8 to $19.7 \%$ at each isolate's MSD (Figs. 1 and 3C-D; Supplementary Fig. S1). Isolates CHCC10, PhPG4, 725Shme, RE18G16, RE18G35, and RE18G26 displayed stimulation in the range of 2.8 to $10 \%$ (Fig. 3C). Isolates 363ShCT18, RE18G38, PhPG22, S088, RE18G8, 500ShCT123, A4, A22, RCC18G15, RE18G4, and D3 exhibited growth stimulation in the range of 10.1 to $19.7 \%$ (Fig. 3D). Four tolerant isolates (PhPG9, 55ShCT173, RE18G45, and H127) did not display significant growth stimulation (Fig. 1 and 3B); the $\gamma$ values for all four isolates were negative, although their TF values ranged from 55.84 to 107.36. There were significant statistical relationships between MSD and NOAEL and between TF and $\mathrm{EC}_{50}$ (Supplementary Fig. S2). No

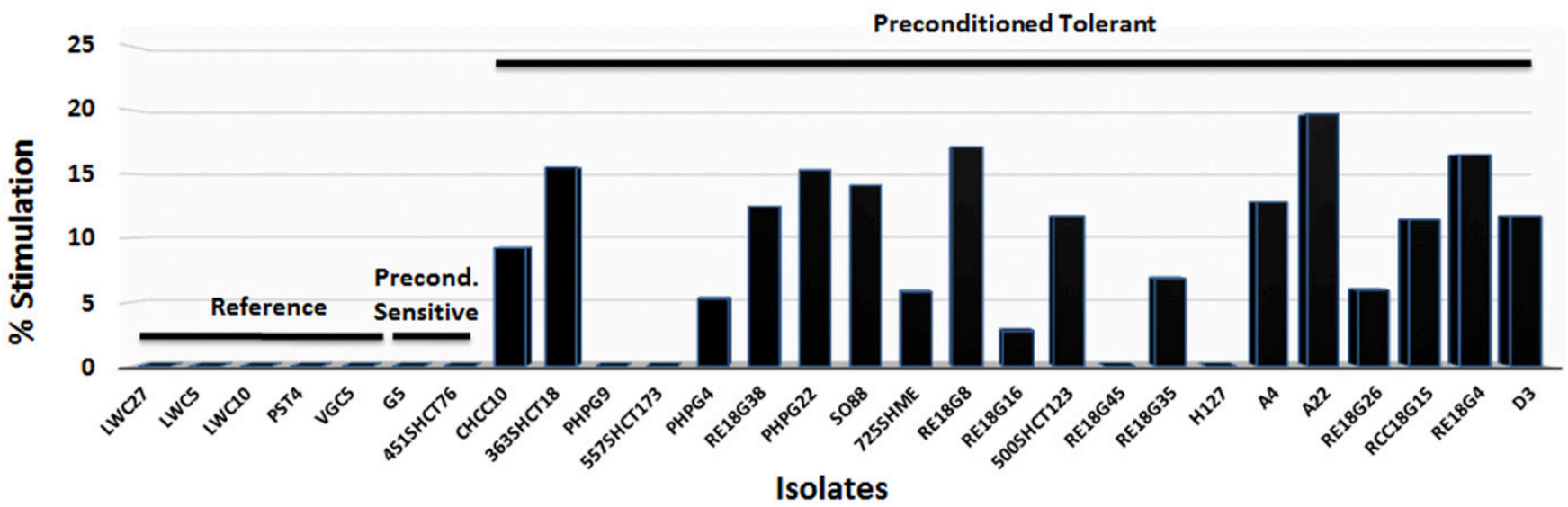

Fig. 1. Twenty-eight Sclerotinia homoeocarpa isolates were analyzed for growth stimulation at subtoxic concentrations of thiophanate-methyl. Isolates were grown on corn meal agar amended with varying fungicide concentrations ranging from 0.047 to $30,500 \mu \mathrm{g} /$ liter and compared with a fungicide-free control. Isolates were grouped according to their tolerance factors in ranges increasing from left to right. The percentage of stimulation was calculated by comparing the growth at the maximum stimulation dose of each isolate with the growth of its corresponding nonamended control. 
significant correlations were found between MSD and $\mathrm{EC}_{50}$ or between TF and NOAEL.

\section{Discussion}

Recurrent exposure to sublethal doses of the fungicide T-methyl preconditioned $S$. homoeocarpa isolates, leading to increased tolerance to T-methyl and hormesis. Twenty-three $S$. homoeocarpa with history of resistance to demethylation inhibitor fungicides and other fungicide classes, but with unknown exposure or documented sensitivity to T-methyl, were grown on T-methyl-amended medium at a

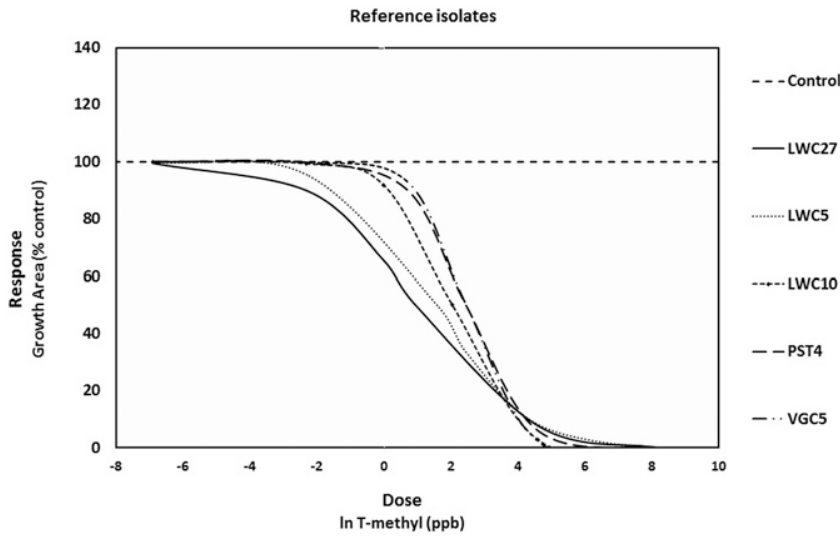

Fig. 2. Pooled modeled curve of five reference isolates of Sclerotinia homoeocarpa in response to thiophanate-methyl (T-methyl) concentrations $(0.047,0.119,0.3,0.75$, $1.89,4.75,11.94,30.5,305,3,050$, and $30,500 \mu \mathrm{g} / \mathrm{liter}$. Three replications were done per concentration, and the experiment was conducted three times for each isolate. concentration 10 times lower than the recommended application rate. These isolates were preconditioned by recurrently transferring mycelial plugs to fresh T-methyl-amended growing medium for 10 cycles. After sensitivity assays were completed, 21 of the preconditioned isolates were classified as tolerant to T-methyl, whereas two were still highly sensitive. Increased mycelial growth when grown on medium amended with subtoxic doses of T-methyl was observed in all the preconditioned isolates compared with the fungicide-free controls; however, significant hormetic responses were observed only in $81 \%$ of them. The five reference isolates, without a known history of fungicide exposure, were highly sensitive to T-methyl, and their growth was not stimulated by sublethal concentrations of T-methyl. These observations indicate that hormesis may be a common response to subtoxic doses of T-methyl in preconditioned $S$. homoeocarpa isolates, although not universal. Nonetheless, the possibility exists that the preconditioned isolates that did not show significant hormesis in vitro may actually respond with virulence stimulation in plant assays, because hormetic stimulation of virulence is often higher in magnitude, and therefore easier to detect, than in vitro growth stimulation (Garzon et al. 2011; Lu et al. 2018). Hence, in vivo testing is necessary to determine the epidemiological relevance of hormesis, and preconditioning hormesis in particular, in this fungal species.

Preconditioning consists of the previous exposure to a low dose of a stressor, which provides protective effects, increasing an organism's tolerance to higher doses of the stressor (Calabrese et al. 2007). Preconditioning also has hormetic effects, which have been described as preconditioning hormesis and have been observed in multiple biological systems (Calabrese 2016a, b). Preconditioning hormesis was recently reported in S. sclerotiorum (Lu et al. 2018) in response to low doses of flusilazole, which resulted in growth
A

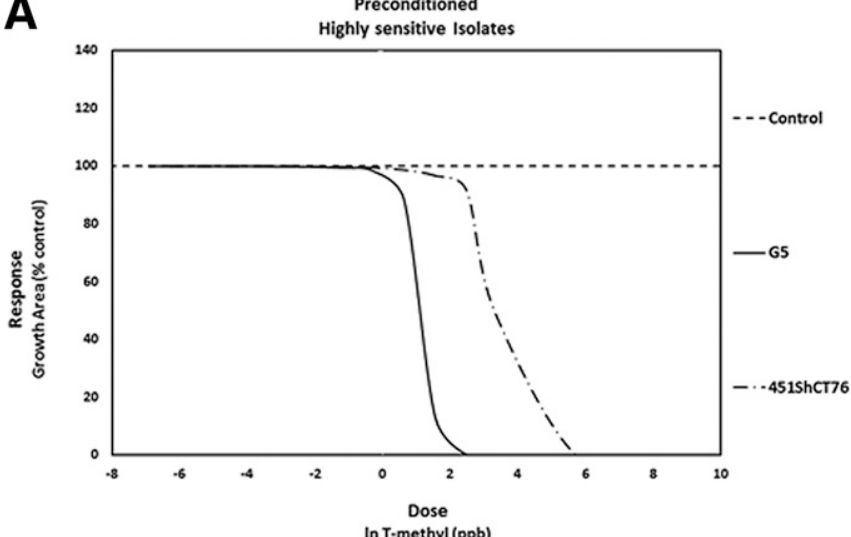

C
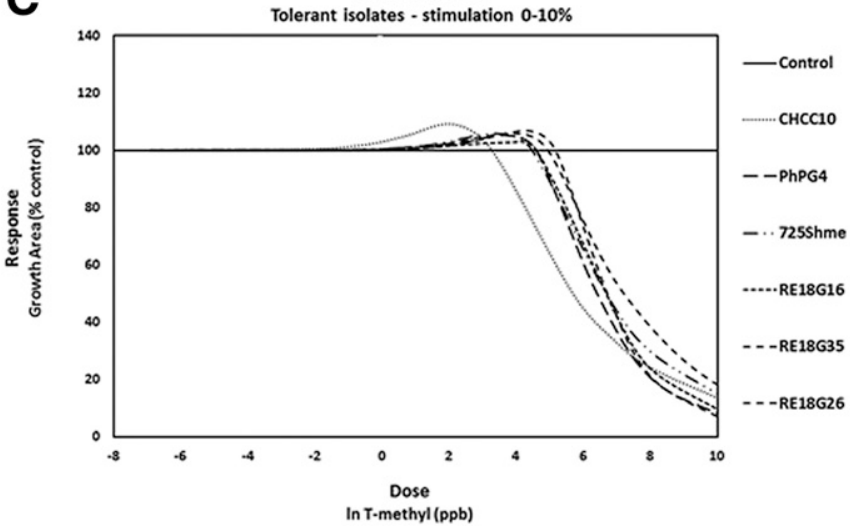

B

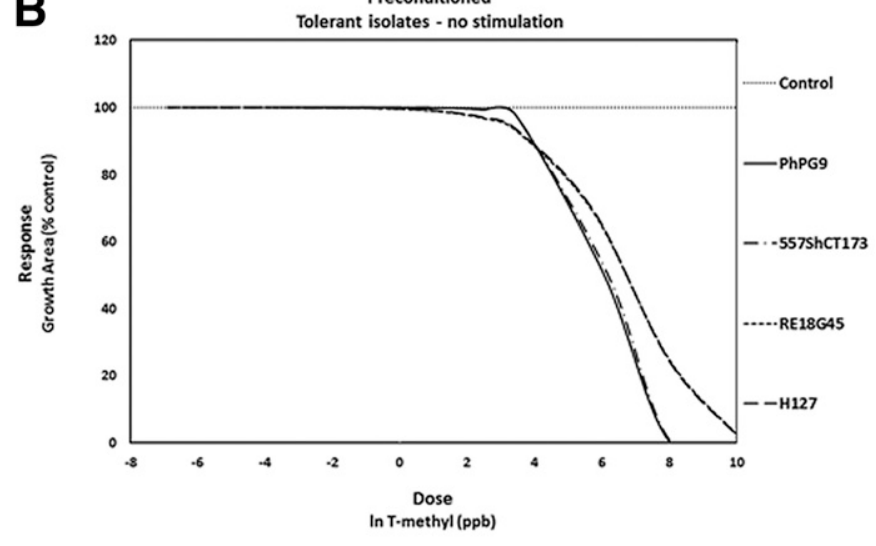

D

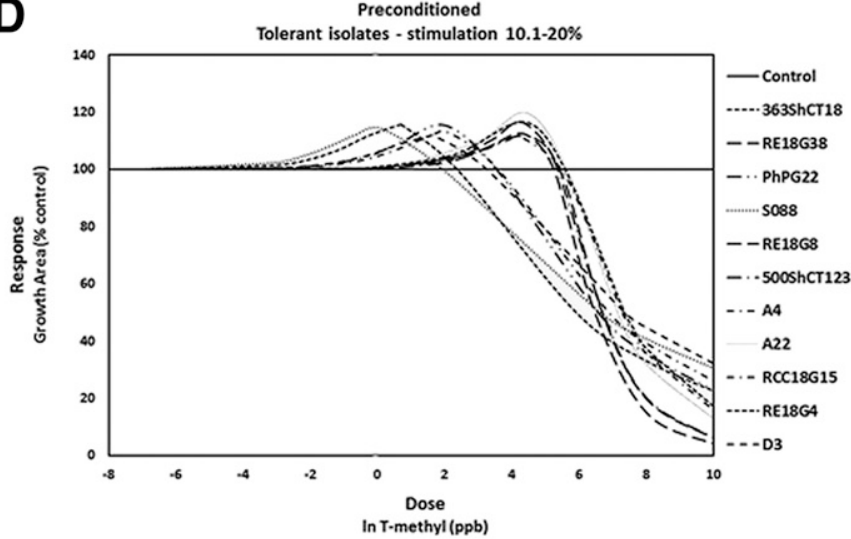

Fig. 3. Pooled modeled curve of preconditioned isolates of Sclerotinia homoeocarpa in response to thiophanate-methyl (T-methyl) concentrations $(0.047,0.119,0.3,0.75,1.89$, 4.75, 11.94, 30.5, 305, 3,050, and 30,500 $\mu \mathrm{g} / \mathrm{liter}$ ): A, preconditioned highly sensitive isolates; $\mathbf{B}$, preconditioned tolerant isolates displaying no significant hormesis; C, preconditioned tolerant isolates displaying stimulation in the range of 1 to $10 \%$; and $\mathbf{D}$, preconditioned tolerant isolates displaying stimulation in the range of 10.1 to $20 \%$. Three replications were done per concentration, and the experiment was conducted three times for each isolate. 
stimulation owing to exposure to hormetic doses of not just flusilazole but also other fungicides with the same mode of action. Further studies are needed to understand the mechanisms involved in fungicide hormesis in general and the crossed effects of preconditioning hormesis to multiple fungicides with the same mode of action in particular.

Several attempts have been made to understand the fundamental mechanism of hormesis. Stebbing (1998) proposed that hormetic responses might result from initial overcorrection at lower concentrations, displayed as strong growth stimulatory peaks, greater than controls. The initial overcorrection subsides gradually at higher concentrations, and the response mechanisms become overwhelmed at the highest concentrations, resulting in inhibition (Stebbing 1998). In this study, the growth stimulation observed in preconditioned isolates might result from an initial overcorrection owing to a disruption in homeostasis, a response most evident in isolates capable of tolerating low doses of T-methyl, even though none of the isolates studied were T-methyl resistant $\left(\mathrm{EC}_{50}<1,000 \mu \mathrm{g}\right.$ of a.i./ml) (Jo et al. 2008). In the case of highly sensitive isolates, the initial overcorrection might not happen at all, or it may be too weak to produce significant stimulation. It may also be possible that highly sensitive isolates may be stimulated at T-methyl concentrations not assayed in this study. Their recovery mechanisms might have become overwhelmed at some of the lowest concentrations. Our findings support the hypothesis that the ability of the fungus to tolerate chemical inhibitors might trigger stress-response mechanisms that result in hormesis. An alternative mechanism proposed by Calabrese involved direct stimulation involving receptors or cell signaling pathways (Calabrese 2013, 2015; Calabrese and Baldwin 2002). Our study did not explore the causes of hormetic responses, but current efforts are focused on identifying metabolic pathways stimulated during hormetic responses.

Calabrese and Blain (2005), based on thorough review of past toxicological studies, developed a hormesis database of approximately 5,600 dose-response relationships, in which more than 900 stressors, including chemicals and physical agents, were used to assess such an effect in a wide range of biological systems (i.e., fungi, bacteria, viruses, plants, and animals, including humans). They concluded that hormesis was broadly generalizable and highly reproducible and that it was a response independent of stressors, biological models, and endpoints (including growth, metabolism, and longevity). Although there is still some disagreement (Mushak 2007, 2013), the consistency of hormesis on a wide array of biological models and endpoints supports its broad generalizability.

Although hormesis is known to be highly reproducible, quantification and assessment of fungicide-induced hormesis in fungi and oomycetes requires standardization of experimental factors including growth media, fungicide concentrations, inoculum developmental stage, incubation temperature, and deliberate testing of several doses below the NOAEL at intervals smaller than one order of magnitude (Garzon and Flores 2013). Crump (2001) suggested strength of evidence, soundness of data, and biological plausibility as the key factors to be considered when evaluating hormesis. Growth stimulation in fungi and oomycetes is often measured by taking two perpendicular diameters of the colony and averaging to obtain a mean of the colony diameter, assuming the colony to be circular (Flores and Garzon 2013; Zhou et al. 2014). However, this technique has some limitations in measuring irregular colonies in which there are significant differences in diameter across replicates, thus affecting measurement accuracy. In this study, we used image measurement software to measure the complete area of the mycelium on solid growing medium, as was done in a previous study on fungicide hormesis of two species of Globisporangium (Pradhan et al. 2017). This method was more reliable and precise for measuring the irregular colonies of S. homoeocarpa.

Past studies have reported the widespread nature of fungicide hormesis in different fungal and oomycete pathogens in vivo (Cong et al. 2018; Di et al. 2015, 2016; Zhou et al. 2014) and in vitro (Baraldi et al. 2003; Flores and Garzon 2013; Pradhan et al. 2017). However, those studies have been conducted using limited numbers of isolates, usually one or a few isolates, that are resistant to a particular class of fungicides. It is still unknown how the different isolates belonging to the same fungal species react to low doses of fungicides. This is the first study to report fungicide hormesis in multiple isolates of $S$. homoeocarpa with varying sensitivity levels to T-methyl. Isolate D3 had the highest tolerance factor but displayed less growth stimulation than other isolates, indicating that higher fungicide tolerance was not directly correlated with higher stimulation at low doses. Because four tolerant isolates did not display significant stimulation, we speculate that fungicide hormesis may be an ability of some, but not all, isolates of this fungal species. Overall, the pooled modeled curve of 28 isolates displayed a wide range of responses for all the T-methyl concentrations, indicating that variation in hormetic responses exists in S. homoeocarpa at an intraspecies level, and possibly in other fungal species. Further molecular studies are necessary to understand the underlying metabolic factors behind the ability to exhibit hormesis in most preconditioned $S$. homoeocarpa isolates.

Although the study of hormetic effects in the field is limited, evidence of fungicide hormesis in a wide range of organisms under laboratory and greenhouse conditions highlights the importance of fungicide hormesis and its potential relevance to plant disease management. Metabolic stimulation in the form of enhanced growth, increased virulence, and higher level of mycotoxin production at low doses of fungicides has been reported in various fungi (Audenaert et al. 2010; Baraldi et al. 2003; Landry et al. 2011; Lu et al. 2018; Zhou et al. 2014) and oomycetes (Flores and Garzon 2013; Garzon et al. 2011; Zhang et al. 1997). More extensive research is required to assess the incidence of hormesis under field and greenhouse conditions as well as to quantify the relevance of fungicide hormesis to crop productivity, particularly in fields or facilities with a history of fungicide resistance in plant pathogenic fungal populations. Hormetic effects may increase pathogen virulence under periods of sublethal fungicide exposure and result in increased disease incidence and severity. It is therefore recommended to consider the concept of fungicide hormesis as part of fungal and oomycete disease management strategies for effective management of crops.

\section{Acknowledgments}

The authors appreciate the contributions of Lane Tredway, Bangya Ma, and Alex Putman for providing $S$. homoeocarpa isolates and histories from their respective collections. We also thank Francisco Flores for his statistical and technical advice in preliminary studies.

\section{Literature Cited}

Audenaert, K., Callewaert, E., Höfte, M., De Saeger, S., and Haesaert, G. 2010. Hydrogen peroxide induced by the fungicide prothioconazole triggers deoxynivalenol (DON) production by Fusarium graminearum. BMC Microbiol. 10:112.

Baraldi, E., Mari, M., Chierici, E., Pondrelli, M., Bertolini, P., and Pratella, G. C. 2003. Studies on thiabendazole resistance of Penicillium expansum of pears: Pathogenic fitness and genetic characterization. Plant Pathol. 52:362-370.

Bennett, F. 1937. Dollar spot disease of turf and its causal organism, Sclerotinia homoeocarpa n. sp. Ann. Appl. Biol. 24:236-257.

Brain, P., and Cousens, R. 1989. An equation to describe dose responses where there is stimulation of growth at low doses. Weed Res. 29:93-96.

Calabrese, E. J. 2013. Hormetic mechanisms. Crit. Rev. Toxicol. 43:580-606.

Calabrese, E. J. 2015. Hormesis within a mechanistic context. Homeopathy 104: 90-96.

Calabrese, E. J. 2016a. Preconditioning is hormesis part I: Documentation, doseresponse features and mechanistic foundations. Pharmacol. Res. 110:242-264.

Calabrese, E. J. 2016b. Preconditioning is hormesis part II: How the conditioning dose mediates protection: Dose optimization within temporal and mechanistic frameworks. Pharmacol. Res. 110:265-275.

Calabrese, E. J., Bachmann, K. A., Bailer, A. J., Bolger, P. M., Borak, J., Cai, L., Cedergreen, N., Cherian, M. G., Chiueh, C. C., and Clarkson, T. W. 2007. Biological stress response terminology: Integrating the concepts of adaptive response and preconditioning stress within a hormetic dose-response framework. Toxicol. Appl. Pharmacol. 222:122-128.

Calabrese, E. J., and Baldwin, L. A. 2002. Defining hormesis. Hum. Exp. Toxicol. 21:91-97.

Calabrese, E. J., and Blain, R. 2005. The occurrence of hormetic dose responses in the toxicological literature, the hormesis database: An overview. Toxicol. Appl. Pharmacol. 202:289-301.

Chen, S. K., Edwards, C. A., and Subler, S. 2001. Effects of the fungicides benomyl, captan and chlorothalonil on soil microbial activity and nitrogen dynamics in laboratory incubations. Soil Biol. Biochem. 33:1971-1980. 
Cole, H., Taylor, B., and Duich, J. 1968. Evidence of differing tolerances to fungicides among isolates of Sclerotinia homoeocarpa. Phytopathology 58: 683-686.

Cong, M., He, S., Ma, H., Li, G., and Zhu, F. 2018. Hormetic effects of carbendazim on the virulence of Botrytis cinerea. Plant Dis. 102:886-891.

Crump, K. 2001. Evaluating the evidence for hormesis: A statistical perspective. Crit. Rev. Toxicol. 31:669-679.

Detweiler, A. R., Vargas, J. M., Jr., and Danneberger, T. K. 1983. Resistance of Sclerotinia homoeocarpa to iprodione and benomyl. Plant Dis. 67:627-630.

Di, Y.-L., Cong, M.-L., Zhang, R., and Zhu, F.-X. 2016. Hormetic effects of trifloxystrobin on aggressiveness of Sclerotinia sclerotiorum. Plant Dis. 100: 2113-2118.

Di, Y. L., Zhu, Z. Q., Lu, X. M., and Zhu, F. X. 2015. Pathogenicity stimulation of Sclerotinia sclerotiorum by subtoxic doses of carbendazim. Plant Dis. 99: 1342-1346.

Flores, F. J., and Garzon, C. D. 2013. Detection and assessment of chemical hormesis on the radial growth in vitro of oomycetes and fungal plant pathogens. Dose Response 11:361-373.

Garzon, C. D., and Flores, F. J. 2013. Hormesis: Biphasic dose-responses to fungicides in plant pathogens and their potential threat to agriculture. Pages 300-328 in: Fungicides: Showcases of Integrated Plant Disease Management from Around the World. M.Nita, ed. IntechOpen, Rijeka, Croatia. DOI:

Garzón, C. D., Molineros, J. E., Yánez, J. M., Flores, F. J., del Mar Jiménez-Gasco, M., and Moorman, G. W. 2011. Sublethal doses of mefenoxam enhance Pythium damping-off of geranium. Plant Dis. 95:1233-1238.

Golembiewski, R. C., Vargas, J. M., Jones, A. L., and Detweiler, A. R. 1995. Detection of demethylation inhibitor (DMI) resistance in Sclerotinia homoeocarpa populations. Plant Dis. 79:491-493.

Hsiang, T., Yang, L., and Barton, W. 1997. Baseline sensitivity and crossresistance to demethylation-inhibiting fungicides in Ontario isolates of Sclerotinia homoeocarpa. Eur. J. Plant Pathol. 103:409-416.

Jo, Y.-K., Chang, S. W., Boehm, M., and Jung, G. 2008. Rapid development of fungicide resistance by Sclerotinia homoeocarpa on turfgrass. Phytopathology 98:1297-1304.

Kato, S., Coe, R., New, L., and Dick, M. W. 1990. Sensitivities of various oomycetes to hymexazol and metalaxyl. J. Gen. Microbiol. 136:2127-2134.

Landry, J., Martinez, C., and Rochefort, L. 2011. The use of fungicide Nova to mitigate infection of Sphagnum by parasitic fungi in the greenhouse. Botany 89:655-661.

Lu, X., He, S., Ma, H., Li, J., and Zhu, F. 2018. Hormetic effects of flusilazole preconditioning on mycelial growth and virulence of Sclerotinia sclerotiorum. Plant Dis. 102:1165-1170.

Ma, B., and Tredway, L. P. 2013. Induced overexpression of cytochrome P450 sterol $14 \alpha$-demethylase gene (CYP51) correlates with sensitivity to demethylation inhibitors (DMIs) in Sclerotinia homoeocarpa. Pest Manage. Sci. 69:1369-1378.
Miller, G. L., Stevenson, K. L., and Burpee, L. L. 2002. Sensitivity of Sclerotinia homoeocarpa isolates to propiconazole and impact on control of dollar spot. Plant Dis. 86:1240-1246.

Monteith, J., Jr., and A. S.Dahl. 1932. Turf diseases and their control. Bull. U.S. Golf Assoc. Green Section 12(4)

Mushak, P. 2007. Hormesis and its place in nonmonotonic dose-response relationships: Some scientific reality checks. Environ. Health Perspect. 115: 500-506.

Mushak, P. 2013. How prevalent is chemical hormesis in the natural and experimental worlds? Sci. Total Environ. 443:573-581.

Nicholson, J., Meyer, W., Sinclair, J., and Butler, J. 1971. Turf isolates of Sclerotinia homoeocarpa tolerant to Dyrene. J. Phytopathol. 72:169-172.

Pradhan, S., Flores, F., Molineros, J. E., Melouk, H., Walker, N. R., and Garzon, C. D. 2017. Improved assessment of mycelial growth stimulation by low doses of mefenoxam in plant pathogenic Globisporangium species. Eur. J. Plant Pathol. 147:477-487.

Putman, A., Carbone, I., and Tredway, L. 2011. Development and characterization of microsatellite markers for Sclerotinia homoeocarpa. Phytopathology 101: S147.

R Core Team. 2013. R: A language and environment for statistical computing. R Foundation for Statistical Computing, Vienna, Austria. http://www.R-project. org/.

Schabenberger, O., Tharp, B. E., Kells, J. J., and Penner, D. 1999. Statistical tests for hormesis and effective dosages in herbicide dose response. Agron. J. 91: 713-721.

Smiley, R. W., Dernoeden, P. H., and Clarke, B. B. 2005. Compendium of Turfgrass Diseases, 3rd Ed. American Phytopathological Society Press, St. Paul, MN.

Stebbing, A. 1998. A theory for growth hormesis. Mutat. Res. 403:249-258.

Stebbing, A. R. D. 1982. Hormesis-The stimulation of growth by low levels of inhibitors. Sci. Total Environ. 22:213-234.

Szabadi, E. 1977. A model of two functionally antagonistic receptor populations activated by the same agonist. J. Theor. Biol. 69:101-112.

Vargas, J. M. 2005. Management of Turfgrass Diseases, 3rd Ed. Wiley, Hoboken, NJ.

Walsh, B., Ikeda, S. S., and Boland, G. J. 1999. Biology and management of dollar spot (Sclerotinia homoeocarpa); an important disease of turfgrass. HortScience 34:13-21.

Warren, C. G., Sanders, P., and Cole, H. 1974. Sclerotinia homoeocarpa tolerance to benzimidazole configuration fungicides. Phytopathology 64:1139-1142.

Zhang, S., Panaccione, D. G., and Gallegly, M. E. 1997. Metalaxyl stimulation of growth of isolates of Phytophthora infestans. Mycologia 89:289-292.

Zhou, F., Liang, H.-J., Di, Y.-L., You, H., and Zhu, F.-X. 2014. Stimulatory effects of sublethal doses of dimethachlon on Sclerotinia sclerotiorum. Plant Dis. 98: $1364-1370$ 